\title{
Techniques for Improving Performance of OFDM System for Wireless Communication
}

\author{
Mr. Umesha G B ${ }^{1}$, Prof. M. N. Shanmukha Swamy ${ }^{2}$ \\ Department of ECE, SJCE, Mysore, Karnataka State, India ${ }^{1,2}$
}

\begin{abstract}
Orthogonal frequency division multiplexing (OFDM) is a special case of multicarrier transmission which transmits a stream of data over a number of lower data rate subcarriers. OFDM splits the total transmission bandwidth into a number of orthogonal and non-overlapping subcarriers and transmit the collection of bits called symbols in parallel using these subcarriers. This works gives a total insight of various Peak -to Average Power Reduction (PAPR) techniques and principles of OFDM systems used in wireless communications. The research work places a focus also on OFDM behaviors and techniques like Carrier Frequency Offset (CFO) estimation that improves performance of OFDM for wireless communications. Finally, this work provides a number of wireless communication standards and many of the applications where OFDM systems are used.
\end{abstract}

Key words: OFDM, Peak-to-Average Power Reduction (PAPR), Inter-Carrier Interference (ICI), Channel estimation, Cyclic Prefix, Bit Error Ratio (BER).

\section{INTRODUCTION}

The recent years has witnessed the demand for higher data rate services such as voice, data and multimedia over both wired and wireless communication links. This demand has increased due to the rapid growth of digital communication systems. The wired and wireless communication environments has been requiring new technologies with new applications like the mobile web access, IP telephony, gaming services, high-definition mobile TV, video conferencing, 3D television and cloud computing. The mobile communication systems like $4 \mathrm{G}$ and $5 \mathrm{G}$ are expected to provide substantially high data rate transmissions in order to meet the requirements of future high performance services such as multimedia, voice, and data applications. OFDM in the $4 \mathrm{G}$ and $5 \mathrm{G}$ community, provide high data rate transmissions with high spectral efficiency, multipath delay speed tolerance, immunity to frequency selective fading channelsand power efficiency. OFDM is a special form of Multi-Carrier Modulation (MCM) technique that was developed in early 1960s. MCM involves splitting the input signal to a number of different signals over one frequency range. Each of these signals are then transmitted, modulated and demodulated over the channel. OFDM was then proposed to be used in wireless applications in 1980s. Due to its reasonable prices of hardware implementations which rely on a very high speed digital signal processing, OFDM has been recognized as an outstanding technique for high-speed data communication systems. The subcarriers in OFDM systems are orthogonal to each other and this orthogonality condition enables the sub-carriers to be closely spaced and overlap without Inter-Carrier Interference (ICI).Although FDMA,TDMA, CDMA are the well-known multiplexing techniques used in wireless communication systems over a number of years, the overall problems encountered in these techniques includes
Inter-Symbol Interference (ISI) resulted from time dispersion, multipath fading, less spectral efficiency and the requirement of larger transmitting power for high-bit rate. OFDM is a very powerful modulation technique which is capable of achieving high data rate transmission without ISI and Inter Carrier Interference (ICI). OFDM is made more efficient by the use of IFFT and its inverse FFT techniques to implement modulation and demodulation functions respectively. The OFDM based wireless communication system design includes the design of OFDM transmitter, and OFDM receiver.

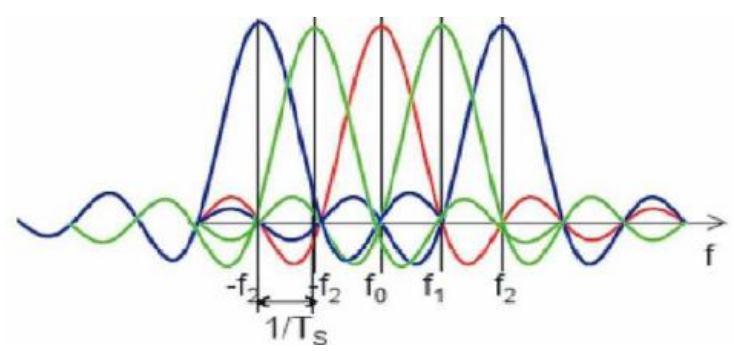

Figure1: OFDM Signal Spectrum

An algorithm in OFDM systems is adopted in the process of generating and receiving sub-carriers by using the digital signal processing technology. However, in order to improve frequency spectrum utilization, each sub-carrier spectrum which meet the orthogonality condition throughout the symbol period(which is longer with narrower bandwidth compared to single-carrier modulation) is overlapped to ensure the receiving end recover the signal without distortion. The OFDM signal spectrum is shown in Figure 1.Techniques for improving the performance of OFDM systems are PAPR reduction and Carrier Frequency Offset (CFO) estimation techniques. 


\section{BASIC PRINCIPAL OF OFDM SYSTEM}

The basic principle of OFDM is to split a high-rate data stream into a number of lower rate streams that aretransmitted simultaneously in parallel over a number of subcarriers. In order to allow for a high spectral efficiency in OFDM systems, the modulation scheme to be used and the carrier power are controlled individually for each carrier. OFDM is generated by choosing the spectrum required based on the input data (stream of bits), and modulation scheme to be used. All the sub-carriers are carefully controlled in order to maintain the orthogonality condition between the carriers. Every sub-carrier OFDM systems is assigned the same data during transmission. As shown in Fig 2, a binary message in form of bits at the transmitter is generated which has a length divisible by the number of subcarriers. The signal of each sub-carrier is first modulated. Each sub-carrier in OFDM system can adopt different modulation scheme such as BPSK, QPSK, 8PSK, 16QAM, 64QAM, etc. This is so depending on thechannel conditions and the need of getting the best balance between the Bit Error Rate (BER) and frequency spectrum utilization.

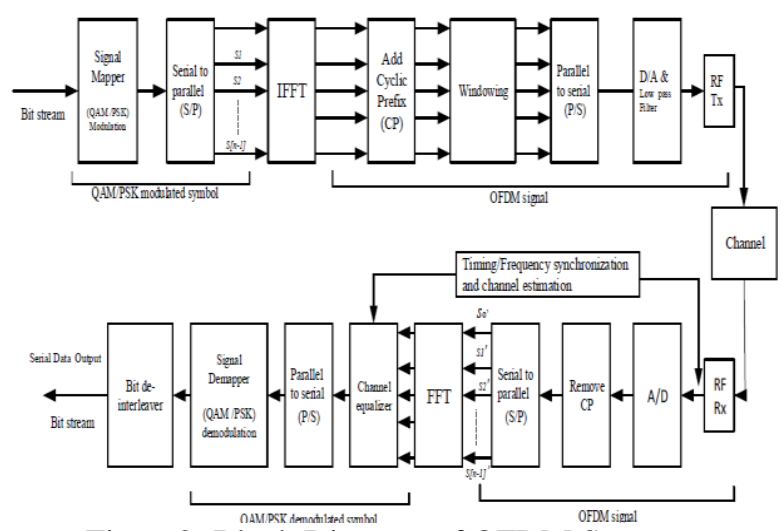

Figure2: Block Diagram of OFDM System

The input serial stream of data is then converted to parallel sets of data at the transmitter. Each set of data in OFDM systems contains one symbol say Snfor every subcarrier. Taking an example, a set of four data set would be [S0, S1, S2, S3].Figure 3 shows how these data set is arranged in the horizontal axis in the frequency domain. The frequency distribution domain of symbols takes the symmetrical arrangement about the vertical axis. This arrangement is necessary for using the Inverse FFT in order to convert the frequency domain data sets into sample data of the corresponding time domain representation.

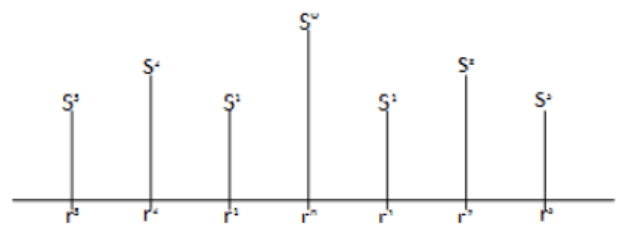

Figure3: Frequency Domain Distribution of Symbols
In OFDM, the IFFT is useful for OFDM system due to the fact that, the samples of a waveform are generated with orthogonal frequency components.

A Cyclic Prefix (CP) is added to each symbol followed by windowing which is a popular method of reducing spectral side lobes in OFDM. Then, the parallel to serial block creates the OFDM signal by sequentially outputting the time domain samples followed also by digital to analog conversion. CP is a periodic extension of the last part of an OFDM symbol.The CP is added to the front of the symbol in the transmitter and then removed at the receiver before the demodulation process is performed. The $\mathrm{CP}$ is inserted in order to convert the linear convolution with the channel impulse response into a cyclic convolution. The cyclic convolution then results into a diagonalised channel that becomes free of both ISI and ICI interference. The CP acts as a guard space between successive OFDM symbols and therefore prevents Inter Symbol Interference (ISI) as long as the length of the CP is longer than the impulse response of the channel. The Cyclic Prefix also ensures orthogonality between the sub-carriers by keeping the OFDM symbol periodic over the extended symbol duration, and therefore avoiding InterCarrier Interference (ICI).

The original data is then recovered from the modulatedsignal through OFDM demodulationtechnique. The received signal pass through a low pass filter and the guard cyclic prefix is removed. The serial data stream is then converted to parallel followed by the FFT of the signal. A demodulator is then used to get back the original signal. By taking the considerations of the un-modulated signal and the data at the receiving end, the bit error rate and the signal to noise ratio is calculated.However, OFDM is constrained by a number of problems also including the Peak-to average power ratio (PAPR), time and frequency synchronization errors which are resulted from the mismatch of oscillators or from the Doppler spread due to relative movement between the receiver and transmitter. Time and frequency varying wireless channels affect the performance of OFDM systems.

Time-varying impairments is the result of theCarrier Frequency Offset (CFO) caused by the mismatch of frequencies between the oscillators at the receiver and transmitter. Frequency-varying impairments is the result of the timing offset or the delay spread of wireless channels. Although OFDM systems are more robust to frequencyselective fading channels but they are more sensitive to the time-frequency varying impairments of channels. These impairments can be completely cancelled in time domain through CFO estimations. TheICI between subcarriers in OFDM systems is introduced by CFO which then destroy the sub-carriers orthogonality and therefore resulting into degradation of the bit error rate (BER).CFO also introduces a phase change proportional to OFDM symbol number as well as the cyclic shift of data between subcarriers. This work focus on different PAPR reduction techniques in OFDM systems. 


\section{TECHNIQUES TO IMPROVE THE PERFORMANCE OF OFDM}

In order to realize the efficiency of OFDM system, it is necessary to justify the performance using different techniques and affecting factors like channel noise, multipath delay spread etc. Techniques to improve the performance of OFDM for wireless communications include Peak-to-Average Power Ratio (PAPR) reduction techniques, channel estimation, time and frequencyvarying impairment mitigation techniques etc. The motivation for PAPR reduction in OFDM systemsto be the nonlinear characteristics of High Power Amplifier (HPA) and Digital-to-Analog Converter (DAC) that can degrade system performance due to induced spectral regrowth and detection inefficiency.

A. Peak to Average power ratio (PAPR) Reduction Technique.

High Peak-to-Average Power Ratio has been recognized as one of the major practical problem involving OFDM modulation. High PAPR results from the nature of the modulation itself where multiple subcarriers or sinusoids are added together to form the signal to be transmitted. PAPR is defined as the ratio of the maximum instantaneous power and the average power, i.e.power occurring in the OFDM transmission to the average power of the OFDM transmission. Mathematically defined as Ppeak $/ \mathrm{P}_{\text {average }}$

$$
P A P R=\underset{0 \leq t \leq T}{\operatorname{Max}} \frac{[x(t)]^{2}}{E\left\{|x(t)|^{2}\right\} \mid}
$$

In order to improve the performance of OFDM in wireless communication systems, several techniques have been proposed in the literature to reduce the PAPR. These techniques are mainly categorized in two parts namely 1) signal Scrambling Techniques 2) signal Distortion Techniques.Signal Scrambling Techniques involve the process of scrambling an input data block of OFDM symbols and transmit the one with a minimum PAPR. In this case, the probability of incurring high PAPR is also significantly reduced.These techniques are further subdivided into two categories that is, techniques which requires explicit side information and techniques which does not need explicit side information. Signal scrambling techniques with side information includes Block Coding Techniques, Selected mapping (SLM), and PartialTransmit Sequence (PTS), Interleaving Technique, Tone Reservation (TR), Tone Injection (TI) and constellation extension techniques.

Signal Distortion Techniques involves the nonlinear saturation around the peaks in order to reduce the PAPR in OFDM systems.The signal distortion techniques can directly reduce high peaks through signal distortion before performing signal amplification. The practical approaches for signal distortion techniques includes clipping and filtering technique, Active constellation,Peak Windowing techniques, Envelope Scaling, Random phase updating, Peak reduction carrier, Compounding, Fourier projection technique, and decision aided reconstruction technique.

\section{SELECTIVE MAPPING TECHNIQUE (SLM)}

Figure 4 shows the block diagram of selective mapping technique for PAPR reduction in OFDM System. In SLM Approach the input data block $\quad \mathrm{X}=\mathrm{x}[0], \mathrm{x}[1], \mathrm{x}[2]$, $\mathrm{x}[3], \ldots \ldots,[\mathrm{N}-1]$ to be transmitted is multiplied by $\mathrm{V}$ different phase sequence

$P^{v}=\left[p_{0}^{v}, p_{1}^{v}, p_{2}^{v} \ldots \ldots p_{N-1}^{v}\right]$

Where $\mathrm{v}=0,1,2,3, \ldots \ldots, \mathrm{N}-1$

Such that the resulting data block becomes of sequence

$$
\mathrm{X}^{\mathrm{v}}=\left\{\mathrm{x}^{\mathrm{v}}[0], \mathrm{x}^{\mathrm{v}}[1], \mathrm{x}^{\mathrm{v}}[2], \ldots \ldots \ldots \ldots . . . \mathrm{x}^{\mathrm{v}}[\mathrm{N}-1]\right\}^{\mathrm{T}}
$$

The signal with the minimum PAPR is then selected for final serial transmission .In fact, when the receiver becomes aware of the predetermined sequence which will enables the minimum PAPR then, the transmitted symbols $\mathrm{X}^{\mathrm{v}}$ can be easily recovered. In SLM the predetermined sequence $\quad X^{v}=\left\{x^{v}[0], x^{v}[1], x^{v}[2], \ldots \ldots \ldots \ldots . . . . . x^{v}[N-\right.$ $1]\}^{\mathrm{T}}$ are known by both transmitter and receiver. Therefore, the information index of the predetermined sequence $\mathrm{P}^{\mathrm{v}}$ needs to be sent to the receiver for each OFDM signal as a side information. However, in order to improve the transmission efficiency, transmission of side information can be omitted by embedding it into an OFDM signal, for example, through a label inserted scrambler and an encoder, or by blindly detecting the optimal phase sequence index in the receiver.

\section{PARTIAL TRANMIT SEQUNCE (PTS)}

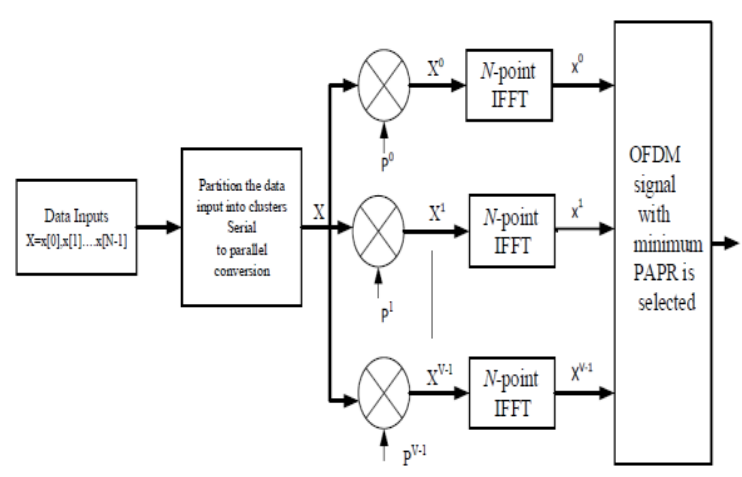

Figure 4: Block Diagram of Selective Mapping (SLM) Technique for PAPR reduction.

The PTS approach is based on partitioning the transmitted OFDM data blocks into several sub-blocks and for each sub-block, multiplying by different weight factors until an optimum value is achieved. As shown in Figure 5, the data information in frequency domain $\mathrm{X}$ of $\mathrm{N}$ symbolsis separated into Vnon-overlapping sub-blocks and each subblock vectors has the same size $X=\left[\mathrm{x}^{0}, \mathrm{x}^{1}, \mathrm{x}^{3}, \ldots . . \mathrm{X}^{\mathrm{v}}\right.$ $\left.{ }^{1}\right]^{T}$,Where $X^{\mathrm{i}}$ represents the sub-blocks that are located 
consecutively to each other.Every partitioned sub-block is the disadvantages associated with clipping technique then multiplied by its corresponding complex phase factor includes, distortion of in-band signal which finally results such that $b^{v}=b^{\mathrm{j} \varphi \mathrm{v}}$, where $\mathrm{v}=1,2,3, \ldots . . \mathrm{V}$. In order to into BER performance and spectral efficiency degradation. obtain the signal in time domain, the IFFT operation is performed on $X^{i}$ to yield the following

$$
X=\operatorname{IFFT}\left\{\sum_{v=1}^{v} b^{v} X^{v}\right\}=\sum_{v=1}^{v} b^{v} \cdot \operatorname{IFFT}\left\{X^{v}\right\}=\sum_{v=1}^{v} b^{v} X^{v}
$$

Another drawback is the out of-band interference signals to adjacent channels caused by out-of-band radiation. The out-of band radiation resulted after performing clipping in the transmitter can be reduced by filtering technique at the cost of peak regrowth. However, filtering cannot reduce in-band distortion. In order to reduce peak regrowth, a The PTS suffers from two main issues which are 1) High computational complexity which is resulted while searching the optimal phase factors.2) The overhead of the optimal phase factors incurred as a side information required to be transmitted to receiver for the correct decoding of the transmitted OFDM symbols. The GA-PTS has lower computational load than original PTS method since it takes advantages of genetic algorithm and simulated annealing algorithm to search the best phase factors of PTS. Clipping is the simplest widely used PAPR reduction technique in OFDM systems. Clipping is mainly performed in the transmitter to limit the maximum of transmit signal to a pre-specified level well as the parts of the signals that are outside the allowed region.

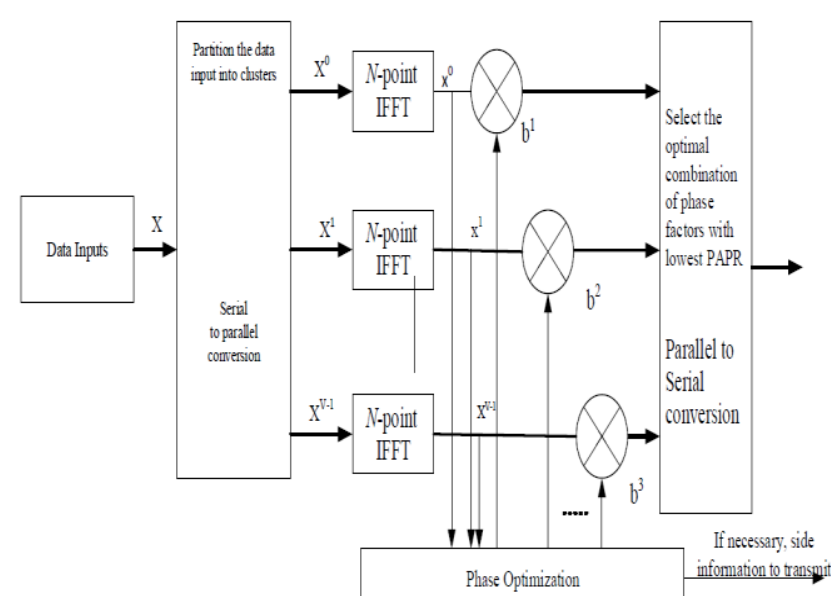
repeated clipping and filtering operation can be used to obtain a desirable PAPR at a cost of computational complexity increase. Figure6 shows the block diagram of PAPR reduction in OFDM systems using clipping and filtering technique. $\mathrm{N}$ represents the total number of subcarriers where $\mathrm{L}$ is the oversampling factor.

\section{CONCLUSION}

The demand for high data rate wireless communication has been increasing dramatically over the last decade. OFDM, which is the most emerging multi-carrier modulation technology of this era, can solve this problem significantly. This work has provided a survey of OFDM behaviors, principles and analyses of different techniques like Peak-to Average Power Ratio (PAPR) reduction and frequency offset estimation that improve performance of OFDM for wireless communications. However, other literature shows that OFDM has a strong anti-multi path interference capability in a high-speed data transfer conditions and have also high spectral efficiency.

\section{REFERENCES}

[1] LIAN Hua, ZhAOruimei, HU boning, PANG Huawei; 2010, Simulation and Analysis of OFDM Communication Systemll, 2010 2nd International Conference on Industrial Mechatronics and Automation. Available in IEEE.

[2] ZHANG jie, L1U liang, LI jin; 2011"Performance Analysis of Space Time Block Code in MIMO-OFDM Systems Available in IEEE.

Figure 5: Block Diagram of PTS algorithm for reducing PAPR

[3] V. Vijayarangan, R. Sukanesh;॥ 2009,An overview of techniques for reducing peak to average power ratio and its selection criteria for orthogonal frequency division multiplexing radio systemsll,Journal of Theoretical and Applied Information Technology.

\section{CLIPPING AND FILTERING}

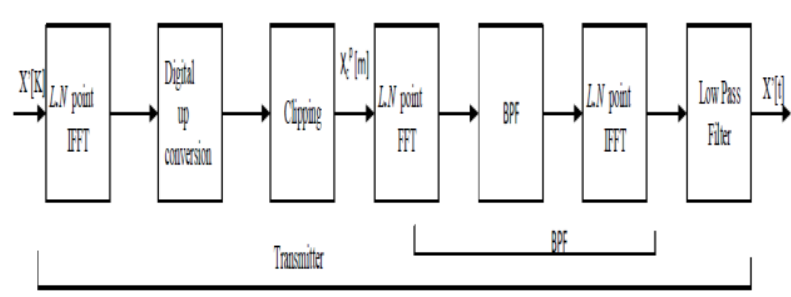

Figure 6: Block Diagram of a PAPR reduction technique using clipping and filtering.

Clipping is the simplest widely used PAPR reduction technique in OFDM systems. Clipping is mainly performed in the transmitter to limit the maximum of transmit signal to a pre-specified level as well as the parts of the signals that are outside the allowed region. Among
[4] Maan Singh, Vijay Kumar; 2013॥Signal Scrambling Techniques for PAPR Reduction in OFDM SystemsInternational Journal of Engineering and Computer Science ISSN: 2319-7242 Volume 2 Issue 1 Jan 2013 Page No. 311-317.

[5] Zhenchuan Zhang, JingjingWu,Study and Performance Simulation Analysis of OFDMSystems", ICWMMN2006 Proceedings.

[6] A.R.S. Bahai, B.R. Saltzberg, multi-carrier digital communications: theory and applications of OFDM, KluwerAcademic/Plenum, Network 1999.

[7] A. Bello, - Selective fading limitations of the Kathryn modem and some system design considerations, „IEEE Trans. Commun.,vol. COM-13, no. 3, pp. 320-333, Sep. 1965.

[8] L. J. Cimini, - Analysis and simulation of a digital mobile channel using orthogonal frequency division multiplexing,॥ IEEE Trans. Commun.,vol. COM-33, no. 7, pp. 665-675, Jul. 1985.

[9] Jyoti Shukla, A lok Joshi, Rahgav Bansal, R.K.Tyagi PAPR Reduction of OFDM systems Using PTS withGenetic Algorithm at low computational complexity,IEEE International Conference on Recent Advances and Innovations in Engineering (ICRAIE-2014), May 09-11, 2014, Jaipur, India. 
[10] Shameema Hameed L, -A Combined Weighting and PTS Technique for PAPR Reduction in OFDM Signalsll, and International Conference on Current Trends in Engineering and Technology, ICCTET'14, IEEE Conference Number - 33344 July 8,2014 , Coimbatore, India.

[11] "IEEE 802.20: Mobile Broadband Wireless Access (MBWA)". Official standards free download web page. IEEE 802 committee. Retrieved August 20, 2011.

[12] Nikookar, H., and Lidsheim, k.S., -Random phase updating algorithm for OFDM transmission with low PAPRI, IEEETransaction on Broadcasting, Vol.48, Jun 2002.

[13] Han, S.H. and Lee, J.H. (2005) an overview of peak-to-average power ratio reduction techniques for multicarrier Transmission. IEEE Wireless Commun., 12(2), 56-65.

[14] Y. Yingwei and G. B. Giannakis, "Blind carrier frequency offset estimation in SISO, MIMO, and multiuser OFDM systems,\| IEEE Trans. Commun., vol. 53, pp. 173-183, Jan. 2005.

[15] S. S. Kim, M. J. Kim and T. A. Gulliver, -PAPR Reduction of OFDM Signals Using Genetic Algorithm PTS Technique,\| IEICE Trans. Communication, vol. E91-B, no.4, pp. 1194-1197, April 2008.

[16] S.-J. Ku, C.-L. Wang, and C.-H. Chen, -A reduced-complexity PTS-based PAPR reduction scheme for OFDM systems, "IEEE Trans. Wireless Commun., vol. 9, no. 8, pp. 2455-2460, Aug. 2010.

[17] S. Younis, A. Al-Dweik1, C. C. Tsimenidis1, B. S. Sharif1 and A. Hazmi(2011), The Effect of Timing Errors on Frequency Offset Estimation in OFDM Systems, IEEE, 2011. 